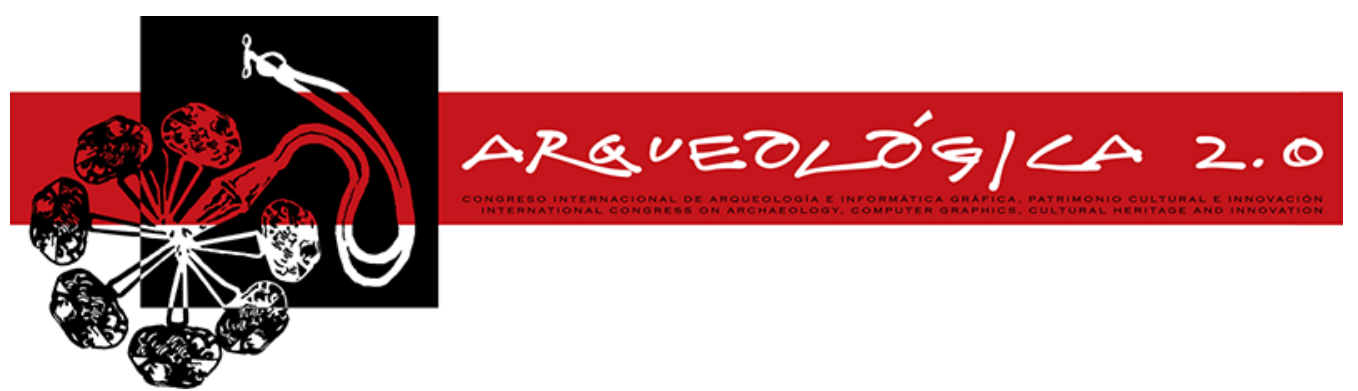

Proceedings of the

$8^{\text {th }}$ International

Congress on

Archaeology,

Computer Graphics,

Cultural Heritage and Innovation

'ARQUEOLÓGICA 2.0'

in Valencia (Spain)

Sept. 5-7, 2016

\title{
EXPLOTACIONES MINERAS EN PATRIMONIO INDUSTRIAL: UN CASO DE USO DE DOCUMENTACIÓN Y SIMULACIÓN
}

\author{
MINING IN INDUSTRIAL HERITAGE: A CASE STUDY OF DOCUMENTATION AND SIMULATION \\ David Marcos $^{\mathrm{a},{ }^{*},}$, José Martínez ${ }^{\mathrm{a}}$, Fco. Javier Delgado ${ }^{\mathrm{b}}$, Javier Finat ${ }^{\mathrm{b}}$ \\ ${ }^{\text {a }}$ Laboratorio de Fotogrametría Arquitectónica, Universidad de Valladolid, Avda. Salamanca s/n, Valladolid, 47140, Spain. \\ Ifa@ega.uva.es; imr@ega.uva.es \\ ${ }^{\text {b } G r u p o ~ M o B i V A P, ~ U n i v e r s i d a d ~ d e ~ V a l l a d o l i d, ~ P a s e o ~ d e ~ B e l e ́ n ~ 11, ~ 47011, ~ S p a i n . ~ j i n a t @ a g t . u v a . e s ~}$
}

\begin{abstract}
:
Mining operations are an essential part of Industrial Heritage. They provide an important reference in order to understand changing past realities, relationships between groups and reconfiguration in the communication between regions whose consequences still remain reference. These realities and relationships have led to the current socio-economic and political framework, which is projected into the future. The documentation of physical vestiges and machinery, now obsolete, is a metaphor that serves to illustrate and understand the past from our present perspective. Threedimensional models from the fusion of different techniques and physical structures contextualization allow to simulatethe mechanisms to promote sustainable tourism as paradigms of a modernity that only serves the immediate appearances. Our approach for documentation and simulation of mechanisms for the extraction and the treatment of mineral is provided as graphical support to understand a reality that goes beyond the "ThematicPark" approach. Moreover, the visualization provides a metaphor for the destruction of natural, physical and human resources of entire areas doomed to depopulation and disappearance. This also opens the door to broader developments that can use multimedia resources to support an all-embracing narrative experience.
\end{abstract}

Keywords: industrial heritage, simulation, 4D reconstruction

\section{Resumen:}

Las explotaciones mineras forman una parte esencial del Patrimonio Industrial. Proporcionan una referencia crucial para entender realidades pretéritas muy cambiantes, relaciones entre dominación entre grupos y reconfiguración en redes de comunicación entre regiones cuyas consecuencias aún perduran. Estas realidades y relaciones han dado lugar a un entramado socioeconómico y político que llega hasta hoy y se proyecta al futuro. La documentación de vestigios físicos y de maquinarias, actualmente obsoletas, son una metáfora que sirve para ilustrar y comprender mejor el pasado y su proyección hacia el futuro desde nuestra perspectiva actual. El modelado 3D de estructuras físicas y su contextualización a partir de la fusión de diferentes técnicas y la simulación de los mecanismos pretenden inicialmente una recuperación museística o impulsar un turismo sostenible como paradigmas de una modernidad que sólo atiende a las apariencias. El enfoque desarrollado para la documentación de instalaciones y simulación de mecanismos para la extracción y el tratamiento del mineral se presenta como soporte gráfico para entender una realidad que va más allá del Parque Temático. La visualización proporciona asimismo una metáfora de la destrucción de los recursos naturales, físicos y humanos de zonas enteras condenadas al despoblamiento y la desaparición, abriendo la puerta a desarrollos más amplios que puedan utilizar recursos multimedia como soporte a una narración más omnicomprensiva.

Palabras clave: patrimonio industrial, simulación, reconstrucción 4D

\section{Introducción}

El Patrimonio Industrial es una de las áreas seleccionadas por la Unesco como emblemática para entender el pasado más reciente vinculado a la Revolución Industrial. La clasificación llevada a cabo en
1999 de forma conjunta por la UNESCO con ICOMOS menciona el Patrimonio Industrial como un área a documentar y proteger, como parte reciente de una memoria colectiva. A finales de los noventa, el Patrimonio Industrial constituía el $5.3 \%$ de sitios culturales y el $4 \%$ de Patrimonio Cultural de la

*Corresponding Author: David Marcos, Ifa@ega.uva.es 
Humanidad $^{1}$; desde entonces, este porcentaje no ha dejado de crecer.

En múltiples ocasiones se ha subrayado que el Patrimonio Industrial no se restringe sólo a los siglos XIX y XX, sino que se remonta a la misma prehistoria. La naturaleza depredadora de la especia humana y la utilización de tecnologías muy destructivas son una constante desde la Antigüedad ${ }^{2}$. En las épocas antigua y medieval y, sobre todo, a partir del Renacimiento, hay una utilización creciente de dispositivos mecánicos para la manufactura que culmina en la primera Revolución Industrial, sin la cual es imposible entender el mundo moderno. Sin embargo, el Patrimonio Industrial suele referirse al período que comienza a finales del s. XVIII y llega aproximadamente hasta 1930.

La rápida obsolescencia de las máquinas iniciales y su sustitución por otras más eficientes, como resultado de la competencia y la disminución de los rendimientos de capital, han dado lugar al abandono o la destrucción de instalaciones, maquinaria y entornos próximos. Esta estrategia responde a una lógica interna depredadores en la que se pretende borrar el pasado (incluso el más reciente), entronizando la inmediatez como si hubiera existido desde siempre.

Asimismo, la desaparición del factor humano en las representaciones documentadas tiene claras consecuencias. De forma implícita, se transmite una representación presuntamente objetiva de un espacio inmutable (como algo dado), el mensaje del carácter inexorable de la destrucción y de la independencia de los procesos con respecto a los agentes que operan sobre ellos. La virtualización de procesos y relaciones, la visualización en la modificación del espacio, la acumulación de capital necesaria para producir los recursos, la representación de los medios técnicos (maquinaria, instalaciones) disponibles en las explotaciones y los papeles desempeñados por los diferentes agentes permiten articular y entender una realidad compleja a partir de vestigios aparentemente inconexos ${ }^{3}$.

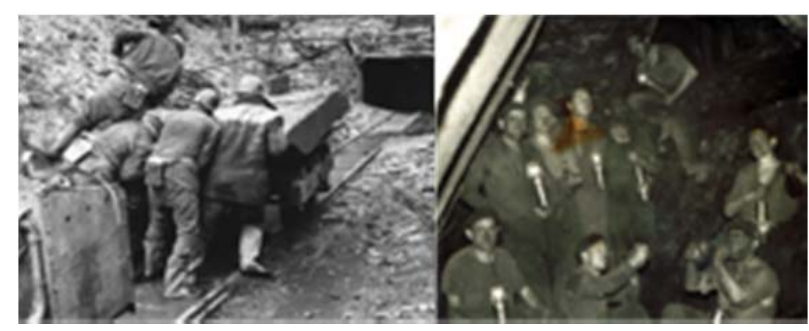

Figura 1: Los mineros como protagonistas del patrimonio industrial.

Nuestra propuesta consiste en que la recuperación del Patrimonio Industrial pasa no sólo por la documentación

Según los datos de World Heritage Site (http://www.worldheritagesite.org).

Un ejemplo extremo bien conocido está dado por la explotación romana de las minas de oro en Las Médulas (Ruina Montium) es el primer paradigma clásico de explotación física, tecnológica y humana a gran escala.

${ }^{3}$ La Ruta de la Plata representa un ejemplo bien conocido de articulación del territorio con unas relaciones sociales cambiantes a lo largo de dos milenios.
3D de instalaciones y la simulación 4D de mecanismos, sino por subrayar el papel y las formas en las que los diferentes agentes de producción (capital, tecnologías, trabajo) se articulan entre sí en un paisaje modificado por la acción del hombre para la producción de mercancías, así como la participación humana en los procesos relacionados en un contexto geográfico y social cambiante. El papel del paisaje hace referencia a una cartografía que va más allá de los aspectos físicos (Maurin 2011)

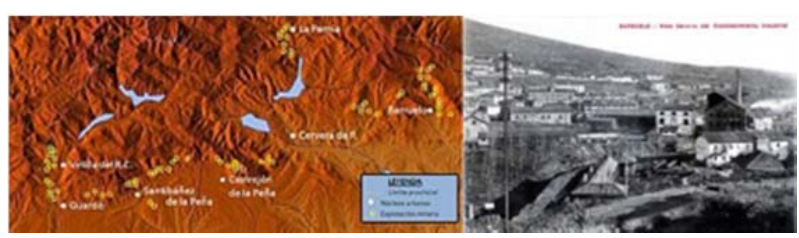

Figura 2: La contextualización geofísica para una comprensión del Patrimonio Industrial.

Por ello, aunque este trabajo esté centrado en la documentación de instalaciones y simulación de maquinaria, debe entenderse como un primer paso para la posterior introducción de un flujo narrativo en el que interactúan diferentes agentes que pueblan y dan sentido a las componentes virtuales y aumentadas en un entorno contextualizado.

En nuestra propuesta, la percepción del paisaje cultural minero requiere una vivencia virtual o real pero adecuadamente contextualizada que reúna elementos gráficos, literales, sonoros, y hasta olfativos que coloquen al visitante en un papel activo en el que pueda percibir que su acción es el motor del cambio, al igual que lo fuera la mano de obra humana incluso en los momentos más inhumanos de la actividad industrial, como se presenta en la Figura 1.

Las tecnologías usadas en la virtualización deben tratar de hacerse imperceptible para ser el instrumento y no el fin (Carrozzino y Bergamasco 2010). Si en el pasado la tecnología minera y sus máquinas camparon en estos paisajes devorando sus recursos y la cultura ancestral transformándolos en otros paisajes con tintes bastante oscuros, hoy debe ayudar a revertir el proceso y contribuir a sacar de la atonía y el subdesarrollo a comarcas enteras que han sido sobreexplotadas hasta perder buena parte de su identidad y su riqueza (Fig. 2).

En una fase posterior, al flujo actual se añadirán avatares (representando a visitantes) que interactúan con personajes (mineros dentro de la aplicación) mediante interfaces de Realidad Aumentada. En el marco virtual actual o en el aumentado por desarrollar, los modelos de realidad virtual o aumentada propuestos para el Patrimonio Industrial permiten recuperar e interpretar el pasado más reciente, al tiempo que proporcionan un soporte ideal para la comprensión de una actividad humana como agentes del cambio, sobre el cual desarrollar recursos narrativos multimedia.

\section{Justificación del enfoque adoptado}

Las explotaciones mineras han sido desde la prehistoria a la actualidad una de las influencias más importantes para el desarrollo de zonas, para el control de recursos y flujos económicos; han determinado variaciones en la distribución de la población concentrándola en los 
núcleos urbanos, han dado pie al desarrollo de industrias locales, y dan explicación a fenómenos migratorios, o a la articulación de redes comerciales entre grupos, regiones o países. Por ello, la documentación de los lugares mineros y técnicas de explotación es uno de los factores más importantes para contextualizar el pasado y entender el presente; en otras palabras, constituye una parte esencial para conocer la Historia, entender la sociedad actual, su evolución desde el pasado hacia el futuro más cercano y las manifestaciones tecnológicas y culturales relacionadas. Para llevar a cabo esta tarea, es necesario contextualizar desde el punto de vista geográfico, histórico, económico y social el espacio físico asociado a las explotaciones mineras y sus transformaciones.

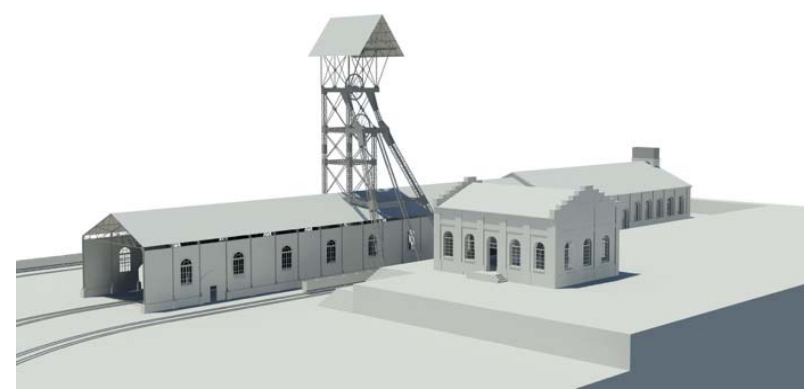

Figura 3: Edificios centrales del Pozo de Herrera.

A la vista del proceso de degradación observado actualmente $y$ de la irreversibilidad de las transformaciones, para recuperar la articulación entre los diferentes aspectos, es necesario llevar a cabo una virtualización de escenarios. Los modelos asociados a esta virtualización proporcionan el soporte para visualizar el entorno y representar una interacción que permita avanzar en el conocimiento de una realidad tan compleja. Para ilustrar esta estrategia hemos desarrollado una documentación y una virtualización del Pozo Herrera dentro de la mina 4 de Sabero situado en la montaña oriental de León (España), cuyos edificios aparecen representados en la Figura 3 y el castillete en la Figura 4.

La labor de documentación del Patrimonio Industrial afecta no sólo a la explotación minera propiamente dicha, sino al entorno y a los agentes que operan sobre él, modificando el paisaje y las relaciones que la especie humana establece con el medio que le rodea y del que forma parte. En la mayor parte de los casos, la relación ha sido y es depredadora, guiada por el principio de maximizar el beneficio en el plazo más breve posible, a costa de la explotación hasta el agotamiento de recursos naturales, físicos y humanos. Esto ha sido así desde épocas históricas antiguas como atestiguan el paisaje de las Médulas explotadas con la técnica de ruina de los montes o las condiciones infrahumanas que se dieron en las iniciales minas de carbón (incluyendo explotación infantil). Tecnologías que sacrificaban todo al objetivo de la producción; medio ambiente, medio físico, cultura y hasta la propia vida.

La extracción del carbón en el Norte de la provincia de Palencia, la parte oriental de la provincia de León y su posterior desplazamiento hacia el Bierzo han dado lugar a profundos cambios en la orografía y las comunicaciones (Sen 1993). Esta extracción presenta diferentes fases de auge y declive asociadas a ciclos económicos tanto financieros (quiebras de mediados del siux y de 1873), como industriales (desmantelamiento de recursos en el Norte de Castilla y León para su concentración en la siderurgia vizcaína). En épocas más recientes también se ha asistido a la deslocalización de recursos entre regiones asociadas a fenómenos estructurales (mala planificación de redes de ferrocarril, yacimientos agotados) o coyunturales (crisis económicas, cambios en las correlaciones de fuerzas de los grupos políticos, ayudas mal utilizadas); la actual fiebre del oro del occidente asturiano o el fracking, son los nuevos paradigmas tecnológicos de una barbarie incesante ligada a la sobreexplotación de toda clase de recursos.

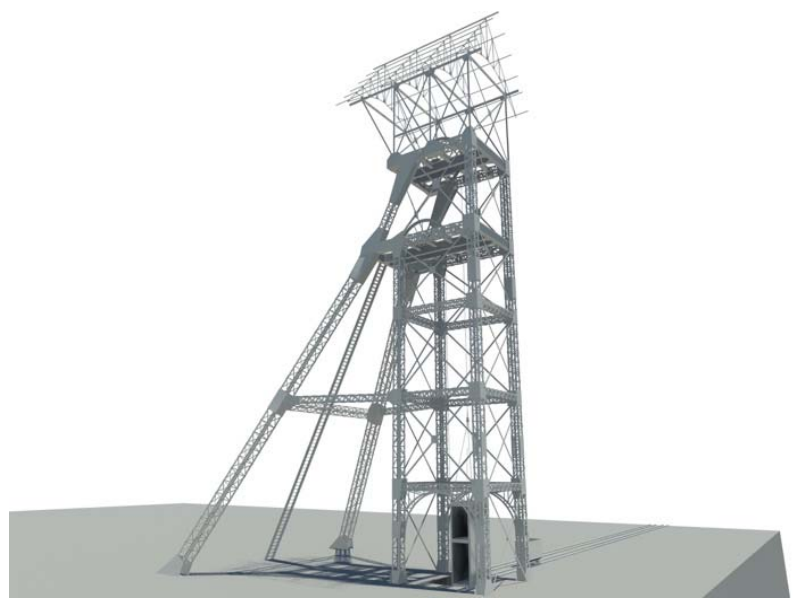

Figura 4: Castillete sobre el brocal del pozo de Herrera.

Lejos de ser una foto fija, la documentación de las explotaciones mineras y su evolución con las estrategias de acumulación originarias reflejan la realidad local, regional y nacional cambiante y, por ello, forman parte de una concepción más amplia del Patrimonio. En (Rabanal 2012) y (Sen 1993) se expone más detalladamente la evolución del sector minero energético que llega hasta finales de los noventa. Estas referencias a la Historia Económica reciente ayudan a comprender la evolución de un sector en crisis casipermanente desde hace un siglo, así como la incapacidad de las políticas institucionales para llevar a cabo una reconversión de la zona actualmente abocada al declive y la despoblación.

A partir de los vestigios físicos representados relacionados con la extracción, es necesario documentar la acumulación de recursos (de capital y tecnológicos), así como la configuración de un tejido socioeconómico que se articula en torno a dichas formas de explotación física, natural y humana. Más allá de una concepción gráfica, la propuesta que desarrollamos consiste en la recreación de una explotación minera como un reflejo de las relaciones sociales y económicas.

Las configuraciones socioeconómicas han cambiado, pero siguen reproduciéndose en la sociedad actual, si bien con una visibilidad y un impacto mucho menor debido a la irrelevancia del subsector minero en el sector primario. Las tecnologías disponibles (vinculadas antes a la Ingeniería de Minas) están al servicio de dichas relaciones $y$, por ello, su documentación y 
visualización gráfica no es un ejercicio aséptico, sino que está vinculado a ilustrar la circulación de mercancías y la reproducción de los recursos utilizados.

El estudio de casos de uso específicos para ilustrar desarrollos de alcance nacional o internacional no es novedoso. En (Vega 2004) se analiza el problema desde un punto de vista más empresarial o sectorial que histórico, complementando el enfoque llevado a cabo por (Rabanal 2012) o (Sen 1993). La visualización gráfica permite acceder a un mayor público y comunicar contenidos académicos o sociales de una forma más. Bajo ningún concepto se pretende ocupar un espacio o reemplazar el trabajo de los profesionales de la Historia Económica reciente, sino proporcionar un soporte que pueda ser reutilizado por expertos procedentes de otras áreas para facilitar una mejor comprensión de entornos olvidados o en declive.

\section{Un caso de uso: el pozo Herrera en Sabero}

La documentación gráfica propuesta utiliza diferentes recursos que permiten enlazar con diferentes aspectos técnicos, históricos o socioeconómicos. Así, por ejemplo, la navegación en las fases iniciales incorpora elementos que pueden ser activados a partir de los cuales se despliegan menús que permiten acceder a dispositivos, modelos y herramientas utilizados para la captura, procesamiento y análisis de la información, incluyendo las diferentes capas superpuestas al objeto virtual o aumentado. De este modo, el soporte gráfico funciona como un referente común a partir del cual se desarrollan diferentes contenidos narrativos que se pueden enlazar al objeto, incluyendo materiales sobre diferentes soportes multimedia.

Este enlazado de contenidos afecta no sólo a recursos tecnológicos, sino también a una intrahistoria de los lugares documentados a partir de los cuales se despliega una historia que ayuda a comprender el pasado durante una visita real o virtual. Las narrativas están vinculadas a objetos digitales que se encuentran embebidos dentro de la aplicación. En particular, el levantamiento arquitectónico permite identificar componentes estructurales y comprender la evolución de la arquitectura civil utilizada en el Patrimonio Industrial.

Por otro lado, los contenidos a insertar a partir de la edición de video están estructurados según diferentes perfiles que pueden ser seleccionados por los usuarios, incluyendo aspectos básicos relativos a las herramientas TIC o a contenidos históricos que facilitan una contextualización de los contenidos mostrados. Un ejemplo típico de estos últimos relevante para la actualidad consiste en mostrar el crecimiento, inicialmente exponencial, de las industrias ligadas a la explotación del carbón. Este crecimiento no es uniforme, sino que está sometido a fuertes vaivenes relacionados con la especulación financiera hacia mediados de los sesenta en el siglo XIX y el consiguiente hundimiento del sector bancario, textil y manufacturero en casi toda la economía castellana.

De forma simultánea y como contrapartida en una economía dual como la española de la época, la prolongación de la industria harinera (como mecanismo de capitalización en mercados interiores) y el crecimiento de las explotaciones mineras (inicialmente en el Norte de Palencia, posteriormente en el Bierzo) proporcionan los principales referentes para repuntes económicos que contribuyen a dinamizar la economía regional. La construcción de líneas locales de ferrocarril para facilitar el traslado del carbón a centros industriales genera una realimentación entre actividades industriales vinculadas al carbón y al hierro que dinamizan regiones emergentes antes de estallar la crisis de 1873. A lo largo del s. XX, la sobre-explotación de recursos hasta el agotamiento de estos últimos, con un valor añadido cada vez más pequeño, unos costes de extracción cada vez más elevados y unos rendimientos decrecientes abocan al cierre de un sector que a finales del s. XIX aportaba más del $40 \%$ de los recursos mineros del territorio nacional (DE VEGA 1995). Los desarrollos vinculados a la transformación se ilustran a partir de la navegación sobre el modelo $3 \mathrm{D}$ de un pequeño horno de siderurgia, modelo sobre el cual se han mapeado las texturas originales.

La sofisticación de los mecanismos de extracción, la percepción actual del carácter esquelético para las construcciones auxiliares, 0 el funcionamiento inexorable (ahora mudo) de una maquinaria (Fig. 5) que trabaja sin descanso hasta agotar todos los recursos son metáforas ilustrativas para entender la sobreexplotación de toda clase de recursos naturales, físicos y humanos. Las construcciones de galerías o el diseño de mecanismos y maquinarias cada vez más complejos son asimismo metáforas, tanto del dominio de la Naturaleza por el hombre -como los ilustrados pretendían hacernos creer- como de una forma de entender la explotación de recursos naturales que, para su efectiva consecución, requiere la explotación de todos los recursos disponibles, incluidos los humanos, a cualquier precio.

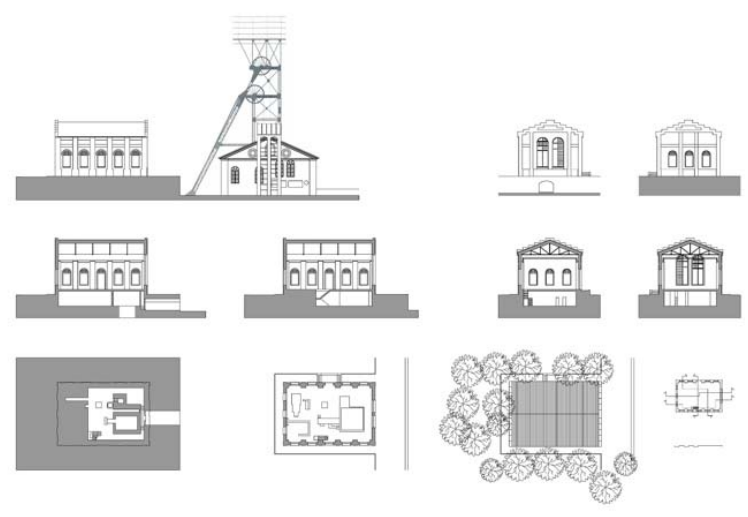

Figura 5: Diferentes planos de la casa de máquinas.

Una representación de los recursos y las tecnologías utilizadas pone de manifiesto que su representación no puede ser neutral. La acumulación de capital para las explotaciones mineras en la segunda mitad del s. XIX procede de los restos de la especulación que conduce a una primera quiebra del sistema financiero en torno a 1864, como prólogo de la general de 1873. Las tecnologías basadas en máquinas de vapor se realimentan del carbón extraído inicialmente en las mismas minas. Sin embargo, el mal diseño de la incipiente red ferroviaria (localización equivocada, tres anchos de vía) lastra el desarrollo inicial de la minería en el Bierzo que no despegará hasta las últimas 
décadas del s. XIX, en detrimento de la hulla palentina (Revilla 1906).

Estos factores muestran un desarrollo desigual de una sociedad dual, pero mantenido durante más de cien años, con importante valor añadido generador de una dinámica completamente diferente a la de la economía basada en rentas agrarias predominante en otras zonas de la Meseta castellana. La concentración de capitales con una dinámica que combina el apalancamiento con la especulación genera empleo en las zonas mineras. Sin embargo, los beneficios son reinvertidos en otras zonas generando un desarrollo desigual y desequilibrado.

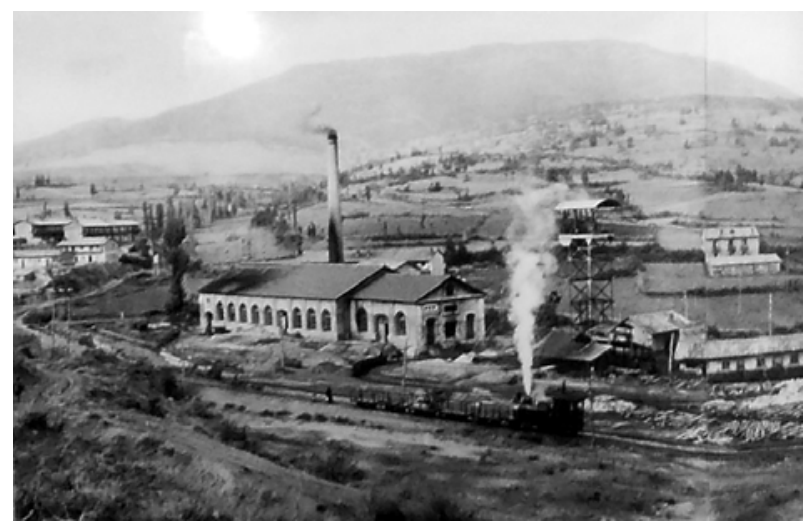

Figura 6: El Pozo de Herrera en los años 30. Años después el castillete fue reemplazado por el actual y la nueva sala de máquinas. La fotografía pertenece al fondo del Museo de la Siderurgia de Sabero.

A medio plazo y a pesar de las infraestructuras generadas, esta dinámica provoca una destrucción del medio. El Museo de la Siderurgia y la Minería de Castilla y León en Sabero (http://www.museosm.com) es una importante iniciativa que trata de recuperar la memoria, a través de diferentes tipos de actividades vinculadas a las antiguas explotaciones mineras, pero aún carece de herramientas para dar soporte multimedia y promover diferentes narraciones superpuestas a diferentes representaciones de un espacio físico. La Figura 6 sirve como ejemplo de los contenidos de este museo.

Esta iniciativa debe motivarse con una comprensión de un medio articulado en torno a explotaciones dispersas. La presencia de maquinarias -muchas herrumbrosas- y equipamientos complejos -algunos peligrosos- quedan como mudos testigos de una dinámica extinta $\mathrm{y}$, como símbolos de la destrucción de un medio, con la imposibilidad de recuperación del tejido social que les daba sentido inicial. La visualización de su funcionamiento es una herramienta útil para comprender la tecnología, pero también para dar una nueva vida a las explotaciones. Para completar este recorrido es necesario desarrollar recursos narrativos en diferentes formatos (texto, imagen, video, representaciones 3D, animaciones) que permitan comprender y proyectar realidades vinculadas al entorno documentado.

En particular, la recuperación del Patrimonio Industrial presenta aspectos relacionados con una dinámica socioeconómica compleja en tejidos urbanos y otros dispersos en explotaciones aisladas. Los primeros se pueden abordar en términos de cambios de uso o reconversiones a espacios culturales y existen diferentes iniciativas en el marco de Proyectos Europeos (como Europa Nostra, p.e.), la reconversión para otros usos (mirador de las estrellas en San Cebrián de Mudá, p.e.) o de iniciativas como la del Museo antes citado en Sabero. Sin embargo y al margen de la recuperación de algunos núcleos para uso vacacional, el despoblamiento de zonas alejadas de los núcleos urbanos, hace realmente difícil una recuperación que vaya más allá del abandono y la constatación del carácter depredador.

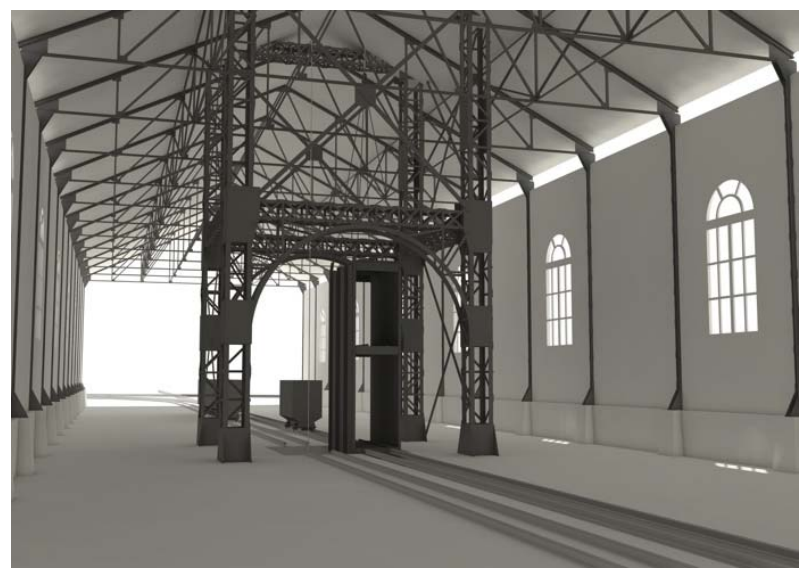

Figura 7: Interior de la nave de vaciado y llenado con base del castillete.

Con estos mimbres, la documentación del Patrimonio Industrial adquiere un carácter completamente diferente del que nos ofrecen declaraciones oficiales o documentos asépticos; tanto los emanados de la Comisión Europea (siempre bienintencionados), como los procedentes de la misma Unesco en relación con el Patrimonio Industrial. Al tiempo que se proclama la necesaria reconversión de zonas que no son rentables, no se ofrece ninguna alternativa real más allá del parque temático. La UE ofrece varios ejemplos de reconversión industrial de zonas mineras deprimidas en polos de producción multimedia en los que colaboran todo tipo de expertos en diferentes tecnologías multimedia y artistas gráficos. La opción del "parque temático" para recuperar zonas arruinadas por el saqueo y la destrucción natural, física y humana tiene un horizonte incierto y por ello es difícil que pueda contribuir a una recuperación del tejido económico y social. Sin embargo, la potenciación del turismo y el desarrollo de recursos narrativos tiene un alcance potencial mucho mayor. Este último involucra a una gran cantidad de agentes que van desde la documentación 3D (como la presentada en este trabajo) hasta la producción de recursos multimedia vinculadas a diferentes soportes.

\section{Localización y contextualización del entorno}

Centrar la atención en los aspectos físicos del entorno, en el triunfo de la tecnología o la generación de una riqueza pasada son otras formas de mixtificación de una realidad muy compleja que las representaciones virtuales o aumentadas pueden contribuir a explicar. EI entorno se ha modificado por la acción del hombre, las tecnologías tienen una vida efímera y la riqueza ha ido a parar siempre muy lejos de las zonas en las que se ha generado. Todos estos aspectos se pueden leer e interpretar a partir de modelos virtuales. Así, por ejemplo: 
a) Las modificaciones sobre el terreno se deben mostrar no sólo sobre Mapas de Elevación Digital para contextualizar la parte visible, sino los sistemas de galerías sobre los cuales se desarrollan las actividades de extracción.

b) La recreación de obras maestras de la ingeniería minera como el canal subterráneo de Orbó $^{4}$ para el transporte de material (1885), o la simulación de las tecnologías utilizadas representadas sobre torres vinculadas a extracción y transporte del mineral.

c) La red ferroviaria articula un espacio económico que facilita la distribución y al cabo del tiempo consolida la desaparición de los recursos por la ausencia de reinversión; un ejemplo paradigmático es el ferrocarril de La Robla construido entre 1889 y 1894 para dar salida al carbón del norte de las provincias de Palencia y León hacia la siderurgia vasca. La utilización posterior en centrales térmicas ha cambiado los recorridos, aunque no los flujos económicos y las actividades vinculadas.

Lamentablemente, las últimas explotaciones que a duras penas sobrevivían han sido cerradas en 2014 , sin apenas otras iniciativas que la creación del Museo de la Siderurgia y la Minería de Castilla y León (Sabero, León) dentro del Plan Sectorial del Patrimonio Industrial incluido en el plan PAHIS (2004-2012). Este cierre condena al ostracismo cuando no a la diáspora a la mayor parte de la población de esas zonas que solo muestran algún signo de vitalidad en los períodos vacacionales. El abandono de las torres y el silencio de los mecanismos que las hicieron funcionar son tan elocuentes de una riqueza evaporada y del carácter depredador como en su día lo fueron de una actividad incesante vinculada a la explotación. La recreación virtual permite mantener viva la memoria relativa a estas actividades, así como una representación de las relaciones que las hicieron posibles. En particular, la recreación virtual de los mecanismos que hacen funcionar los dispositivos adquiere múltiples sentidos que va más allá de la apariencia física o de las tecnologías (máquinas de vapor, electricidad) que las mueven.

En este trabajo hemos analizado y modelado la mina del Pozo Herrera. El levantamiento $3 \mathrm{D}$ ha sido llevado a cabo de forma híbrida, es decir, combinando escáner láser y fotogrametría convencional.

La captura de datos del complejo minero y de su entorno se ha realizado mediante escaneos 3D con un escáner de la marca Optech IIris 3D. Se trata de un escáner de rango de medición de tiempo de vuelo de largo alcance que permite escaneos a una distancia de un kilómetro con una precisión centimétrica. Su elección está justificada debido a la complejidad del entorno, ya que los edificios se encuentran ubicados en un valle y con este escáner podemos obtener mediciones precisas desde posiciones elevadas a cierta distancia capturando zonas amplias del complejo. Para este trabajo se han escaneado tanto el exterior como el interior del complejo (nave de llenado, oficinas, vestuarios, salas de

4www.arqueologiaypatrimonioindustrial.com/2008/12/minas-deorb-el-canal-subterraneo.html máquinas, etc.). Desde la posición de cada tomase han realizado mediciones radiométricas (color) mediante fotografías panorámicas que serán utilizadas para colorear las nubes de puntos en el post-procesamiento.

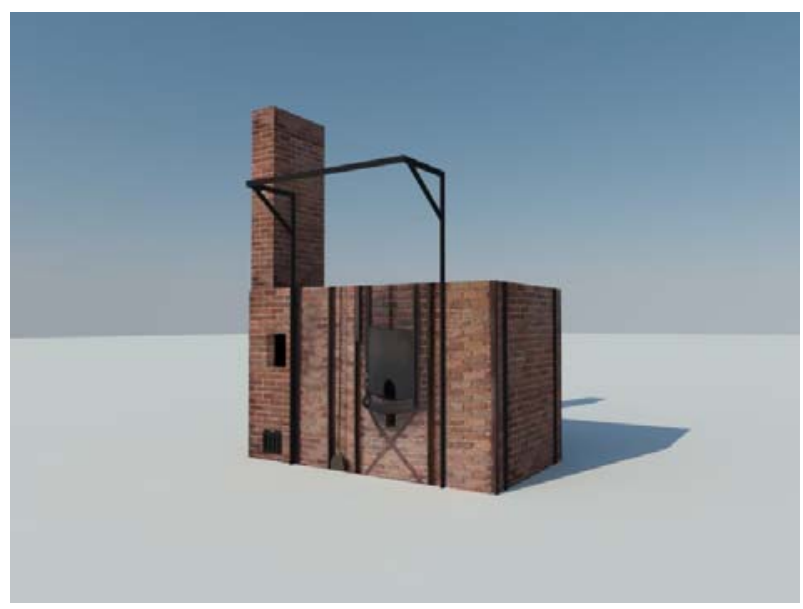

Figura 8: Pequeño horno de siderurgia en las instalaciones anexas

Además de los escaneos, el complejo ha sido digitalizado con fotografía de alta resolución, utilizando una cámara DSLR. Estas capturas han incluido vistas detalladas de todos los elementos y espacios significativos que más tarde han servido como texturas durante el modelado 3D. La Figura 8 ilustra la generación de un modelo $3 \mathrm{D}$ con texturas para representar un horno de siderurgia. Estas fotografías han sido combinadas con panorámicas equirectangulares esféricas de espacios significativos por su relevancia histórica, con la intención de mostrar el estado actual del edificio en los nuevos dispositivos de realidad virtual y en otras aplicaciones.

El procesado de los datos obtenidos con el escáner permite construir una única nube de puntos del complejo de edificios y de su entorno. A su vez, este modelo proporciona un soporte 3D para la obtención de ortoalzados y secciones 2D (perfiles) conformadas mediante proyección de puntos seleccionados sobre planos. Para este proceso se ha utilizado UVaCAD®, un software propio creado por el LFA-DAVAP de la Universidad de Valladolid. A partir de estas representaciones planas se vuelve a una tridimensionalidad generalizada 0 simplificada, construida a base de primitivas y operaciones de diseño asistido por computador, tales como extrusión, simetría, etc. Esta etapa de simplificación fue llevada a cabo con el software Autodesk AutoCAD®.

La reconstrucción de la maquinaria ha sido llevada a cabo siguiendo las mediciones realizadas con el escáner láser y las fotografías, fusionando la información con el tratado de maquinarias de (Flachat 1842). Una vez conseguido un modelo con una complejidad geométrica aceptable, se han diseñado y reconstruido las complejas estructuras mecánicas de las torres vinculadas a la extracción del mineral y las edificaciones que alojaban la actividad minera o industrias auxiliares. En esta etapa se han diseñado nuevos materiales para el modelo 3D, así como animaciones mediante la técnica de fotogramas clave o keyframes. El trabajo consiste en determinar las 
acciones que se desencadenarán en esos fotogramas clave, para que el software de animación sea capaz de determinar el valor de las variables por interpolación entre frames.

El último paso ha consistido en generar un vídeo con una parte estática en la que se describen los elementos más significativos del entorno del pozo que pueden ser visitados de forma segura y que se describen a continuación.

1. La sala de máquinas, en cuyo interior estaba el motor diésel que movía el cable para subir y bajar los ascensores.

2. El castillete o torre utilizada para la extracción del material mediante un sistema de poleas sobre el brocal del pozo. La extracción del material (sobre trenes de vagones), o el desplazamiento de personas, se llevaban a cabo mediante izado vertical utilizando cabrestantes, que pueden incluir castilletes para izado/bajada y mejorar la seguridad de los trabajadores (Fig. 4).

3. La nave de llenado y vaciado, con dos vías para la entrada y salida de vagonetas (vacías o llenas de carbón, respectivamente), como aparece en la Figura 7.

4. Las vías, que aparecen resaltadas para indicar el flujo de trabajo hacia el interior de la mina con vagonetas inicialmente vacías que se bajaban utilizando la maquinaria del castillete para ser cargadas por los mineros.

5. Las vías de vaciado, que salen de la nave de llenado o vaciado, cierran el ciclo básico asociado a la extracción del material en relación con las vagonetas procedentes del interior de la mina, una vez cargadas.

6. Una nave anexa que contenía las salas de vestuarios, duchas y una enfermería.

7. Una serrería (parte izquierda del vídeo) en la que se realizaban las tareas necesarias para fabricar elementos que permitan consolidar la estructura interna de las minas (entibaciones).

La segunda parte del video es dinámica, y muestra una navegación visual alrededor de las instalaciones y en el interior del pozo mediante una cámara virtual. Del interior se muestran detalles de la arquitectura civil, de las instalaciones industriales y de la maquinaria, incluyendo una recreación de su funcionamiento.

\section{Retos y trabajo futuro}

Uno de los principales retos abordados en este proyecto es la eliminación de los obstáculos físicos para la visita y el reconocimiento de estos paisajes. La virtualización del patrimonio ha de convertirse en el medio por excelencia para la superación de todas las limitaciones. La adopción de estas tecnologías por el sector turístico debe saber sacar partido a los recursos que hoy permiten ampliar el espectro de potenciales visitantes a todas las edades y condiciones físicas. La revitalización del sector será clave para la creación de polos de atracción y la generación de empleo en toda la cadena: productores de contenidos, servicios de gestión, desarrollo de herramientas de realidad virtual $y$ aumentada, agentes y gestores de turismo, hostelería, comercio etc.

Esto conlleva además otro reto añadido asociado a la necesidad de incrementar las habilidades, capacitación y profesionalización de los agentes culturales y turísticos involucrados alrededor de este tipo de contenidos, que a su vez debería traer consigo el acercamiento y la participación de las empresas de tecnología en áreas geográficas que hoy se encuentran en un proceso de desvitalización que es necesario atajar. Así se pueden promover líneas negocio en sectores culturales ligados a la arqueología y el patrimonio industrial necesitados de inversores privados. Es necesario facilitar la difusión internacional de los paisajes culturales en general, y mineros en particular, con su inclusión en redes europeas para que formen parte de una memoria que tiene elementos dignos de ser conservados y que forman parte de nuestra identidad cultural.

En un trabajo que todavía está en ciernes se distinguen algunas de las posibles líneas de trabajo futuro. Una de ellas consiste en la posibilidad de seleccionar de forma interactiva los modelos digitales con zonas o regiones que pueden ser seleccionadas de forma interactiva para componer relatos. Los diferentes usuarios podrán insertar sus experiencias y conocimientos en forma de diferentes recursos narrativos (geológicos, históricos, literarios, fílmicos) con los que ayuden a preservar el patrimonio al mismo tiempo que se fomenta la participación en la memoria colectiva.

Otra posible línea de trabajo consiste en incorporar agentes humanos vinculados a las explotaciones mineras y que hagan funcionar los dispositivos. El esquema para incorporar estos agentes se plantea en términos de herramientas avanzadas para la interacción (usando herramientas de Second Life, p.e.) o adaptando estrategias de videojuegos. La ausencia actual de figuras humanas en estas simulaciones es otra mistificación de la técnica, como si pudiera funcionar más allá de los agentes que las utilizan, como si la explotación de los recursos físicos a través de los tecnológicos no se pudiera hacer sin la explotación de los recursos humanos, invisibles en las representaciones asociadas a la documentación. Por ello, es necesario incorporar a diferentes agentes humanos en la visualización del Patrimonio Industrial, como actividad de transformación. Por el momento, los desarrollos se han centrado en la virtualización del entorno y en la simulación de las técnicas que hacen funcionar los diferentes dispositivos mecánicos asociados a la extracción.

\section{Conclusiones}

Este trabajo presenta una primera aproximación al levantamiento fotogramétrico, modelado 3D y simulación del caso de estudio del Pozo de Herrera de las minas de Sabero como ejemplo del Patrimonio Industrial en explotaciones mineras. El esquema desarrollado en esta propuesta parte de la representación gráfica como un pretexto a partir del cual se desarrollan diferentes narrativas vinculadas a actividades humanas. El resultado final ha sido un video en el que una cámara virtual recorre las instalaciones mientras se reproduce la simulación. Al igual que en otras aplicaciones del Patrimonio Histórico, los modelos 3D diseñados nos 
proporcionan un soporte digital para la producción de contenidos multimedia adicionales, pudiendo incorporar investigaciones recientes sobre el desarrollo regional, productivo vinculado a la minería y socioeconómico.

\section{Agradecimientos}

Este trabajo ha formado parte del proyecto "Inventario y Documentación del Patrimonio Histórico Industrial de la provincia de León" (número de expediente 01360/200966), financiado por la Dirección General de Patrimonio de la Junta de Castilla y León. Igualmente, los autores agradecen las facilidades prestadas por la Asociación Llámpara y el Museo de La Siderurgia y La Minería de Castilla y León (Sabero, León) para el acceso y la recopilación de la documentación histórica necesaria.

\section{Referencias}

CARROZZINO, M. y BERGAMASCO, M. 2010. Beyond virtual museums: Experiencing immersive virtual reality in real museums. Journal of Cultural Heritage, 11(4), pp 452-458.

DE VEGA, M.E., 1995. El sueño imposible de una burguesía agraria. Historia de una Cultura (las castillas que no fueron). Sever-Cuesta. 1(3), pp. 323-359. ISBN 84-7846-462-X

FLACHAT, E., BARRAULT, A. ET PÉTIET, J., 1842. Traité de la fabrication du fer et de la fonte envisagée sous les rapports chimique, mécanique et commercial. $D$ Avanzo et cie.

MAURIN ALVAREZ, M., 2011: Huella, memoria y patrimonio territorial de la minería española. Una síntesis cartográfica. Ería 86, pp. 187-214. Available: https://dialnet.unirioja.es/descarga/articulo/3819919.pdf [1/06, 2016]

RABANAL, N.G., 2012: El sector minero energético. In: XXV años de Economistas y Economía Leonesa. Colegio de Economistas de León, pp. 63-90.

REVILLA HAYA, J., 1906: Riqueza minera de la provincia de León. Su descripción, industria y estudio de soluciones para explotarla. Madrid, Imprenta Alemana. Suplemento $1^{\circ}, 48$ pages.

SEN RODRÍGUEZ, L.C. 1993: La minería leonesa del carbón, 1764-1959: una historia económica. Universidad de León. Secretariado de Publicaciones y Medios Audiovisuales. 371 pages. ISBN 84-7719-364-9.

VEGA CRESPO, J., 2004: Minería y desarrollo empresarial en León: el caso de la minero-siderúrgica de Ponferrada. Departamento de Economía Aplicada, Universidad de Valladolid. Available: http://www.usc.es/estaticos/congresos/histec05/b16 vega crespo.pdf [1/06, 2016] 